\title{
Infrared Absorbing Croconaine Dyes: Synthesis and Metal Ion Binding Properties
}

\author{
Rekha R. Avirah, Kuthanapillil Jyothish and Danaboyina Ramaiah* \\ Photosciences and Photonics, Chemical Sciences and Technology Division \\ National Institute for Interdisciplinary Science and Technology (NIIST) \\ CSIR, Trivandrum, INDIA 695019 \\ Tel: +914712515362 \\ Fax: +914712490186 \\ E-mail : rama@csrrltrd.ren.nic.in or d_ramaiah@ rediffmail.com
}

* To whom correspondence should be addressed:

\section{Supporting Information}

\section{Contents}

General Methods

${ }^{1} \mathrm{H}$ and ${ }^{13} \mathrm{C}$ NMR spectra of the representative quinaldinium salts and croconaine dyes (Figures S1-S7)

Absorption properties of the croconaine dyes 3a-d (Table S1)

Changes in absorption and emission spectra of the dye $\mathbf{3 d}$ with the addition of representative metal ions (Figures S8- S9)

\section{Page No.}

$\mathrm{S} 2$

S3

S6

S7 


\section{General Methods}

The equipment and procedure for spectral recordings are described elsewhere. ${ }^{1}$ All the solvents used were purified and distilled before use. Metal ion binding studies were carried out by the addition of equal aliquots of metal ion stock solution in water to $3 \mathrm{~mL}$ of dye in THF.

Starting Materials. 6-Bromo-2-quinaldine (1b) $\left(\mathrm{mp} 95-96{ }^{0} \mathrm{C}\right),{ }^{2}$ 6-iodo-2-quinaldine (1c) $\left(\mathrm{mp} 108-109{ }^{0} \mathrm{C}\right),{ }^{2}$ cholester-3-yl-2-methylquinoline-6-yl carbonate $(\mathbf{1 d})\left(\mathrm{mp} 101-102{ }^{0} \mathrm{C}\right),{ }^{3} \mathrm{~N}-$ methyl-2-quinaldinium iodide (2a) (mp 195-196 $\left.{ }^{0} \mathrm{C}\right),{ }^{4}$ 6-bromo-N-methyl-2-quinaldinium iodide (2b) $\left(\mathrm{mp} 237{ }^{0} \mathrm{C}\right),{ }^{2}$ 6-iodo-N-methyl-2-quinaldinium iodide (2c) (mp 222-223 $\left.{ }^{0} \mathrm{C}\right),{ }^{2}$ and cholester-3-yl-N-methyl-2-quinaldinium-6-yl carbonate iodide (2d) (mp 216-217 $\left.{ }^{0} \mathrm{C}\right)^{3}$ were prepared by modifying the reported procedures.

\section{References}

1) (a) Joseph, J.; Eldho, N. V.; Ramaiah, D. Chem. Eur. J. 2003, 9, 5926. (b) Joseph, J.; Eldho, N. V.; Ramaiah, D. J. Phys. Chem. B 2003, 107, 4444. (c) Kuruvilla, E.; Joseph, J.; Ramaiah, D. J. Phys. Chem. B 2005, 109, 21997.

2) Jha, B. N.; Banerji, J. C. Dyes and Pigments, 1983, 4, 77.

3) Jyothish, K.; Avirah, R. R.; Ramaiah, D. Org. Lett. 2006, 8, 111-114.

4) Doebner, A.; von Miller, W. Ber. 1883, 16, 2464. 


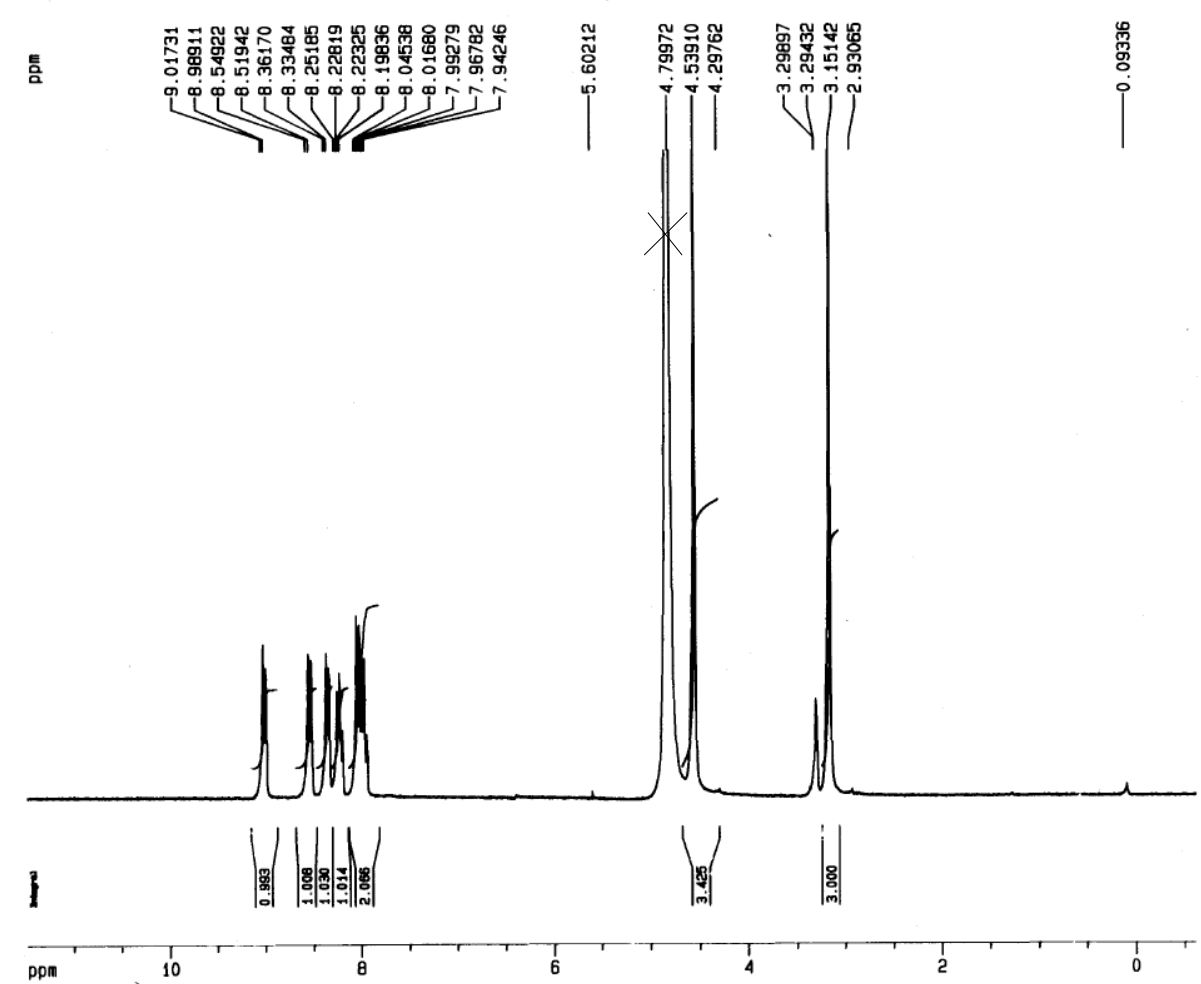

Figure S1. ${ }^{1} \mathrm{H}$ NMR spectrum of the quinaldine salt $\mathbf{2 a}$ in DMSO-d $\mathrm{d}_{6}$.

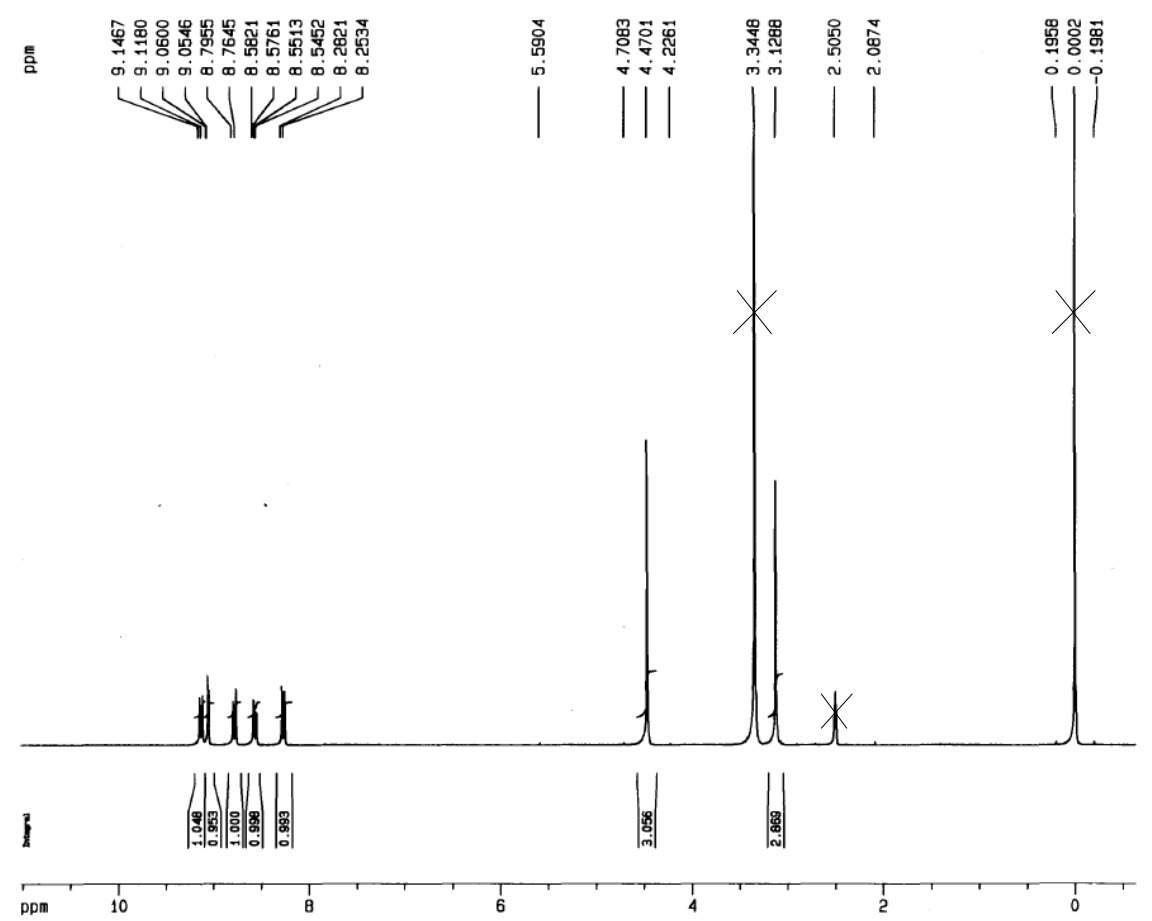

Figure S2. ${ }^{1} \mathrm{H}$ NMR spectrum of the quinaldine salt $\mathbf{2 b}$ in $\mathrm{MeOH}-\mathrm{d}_{4}$. 


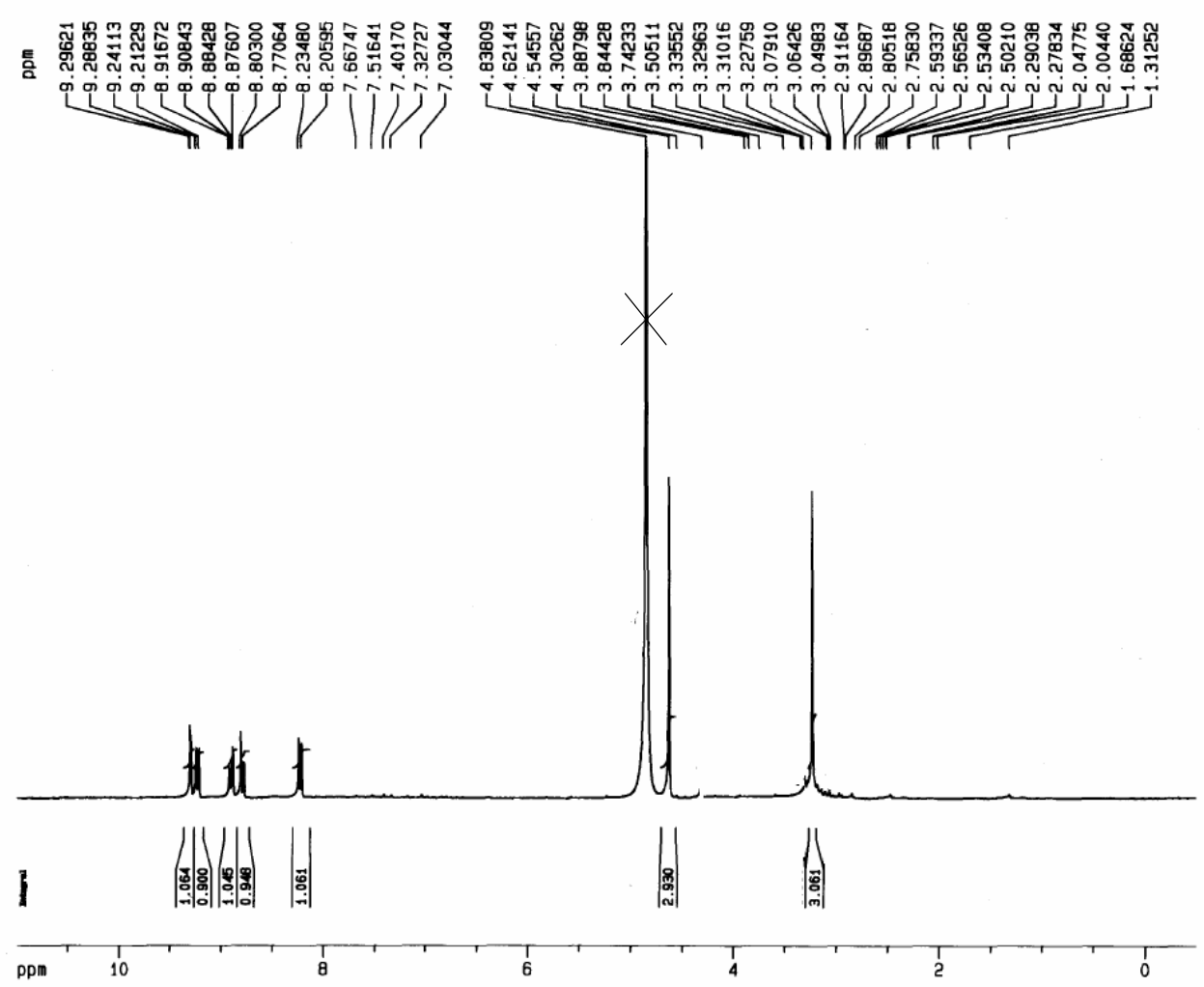

Figure S3. ${ }^{1} \mathrm{H}$ NMR spectrum of the quinaldine salt $2 \mathbf{c}$ in DMSO- $\mathrm{d}_{6}$.

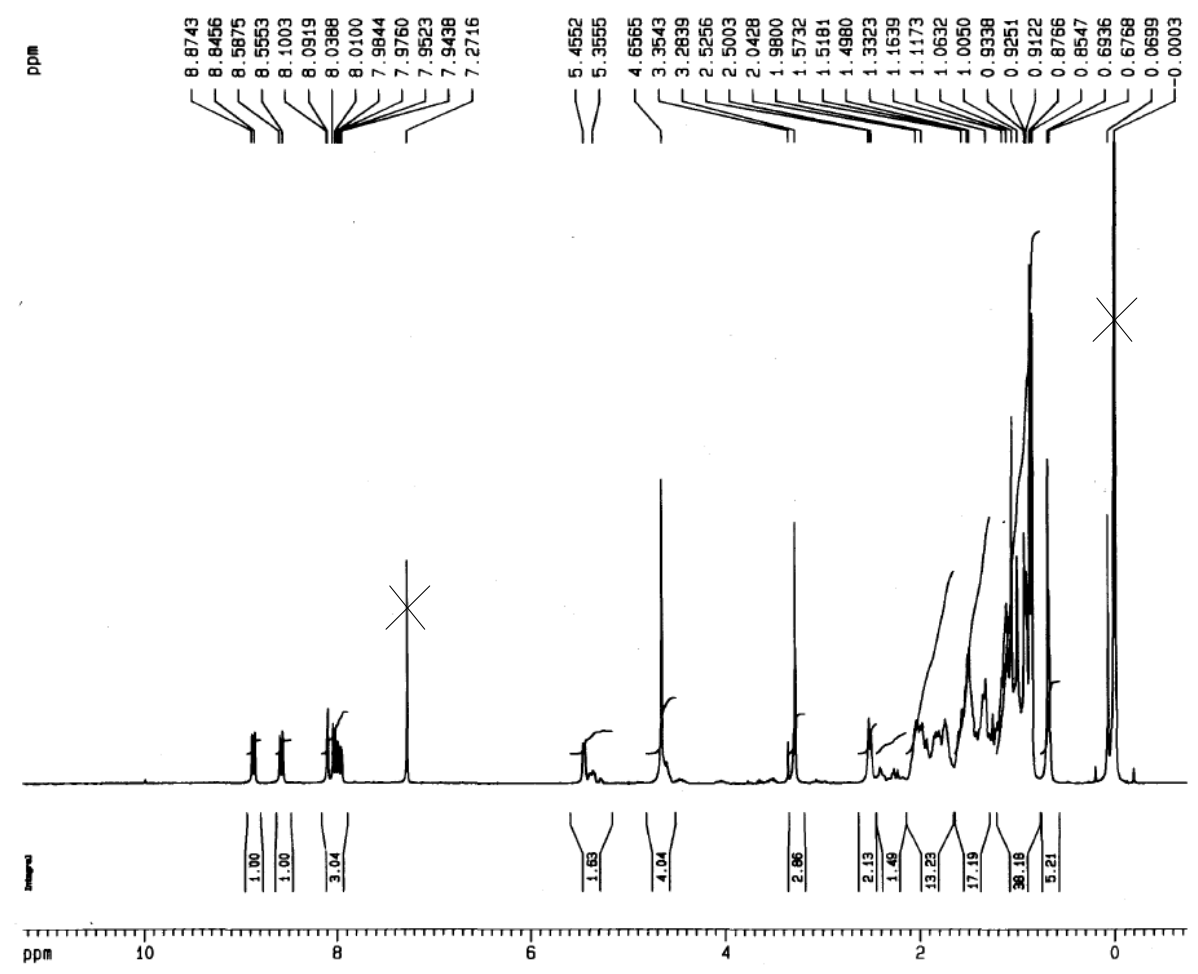

Figure S4. ${ }^{1} \mathrm{H}$ NMR spectrum of the quinaldine salt $\mathbf{2 d}$ in $\mathrm{CDCl}_{3}$. 


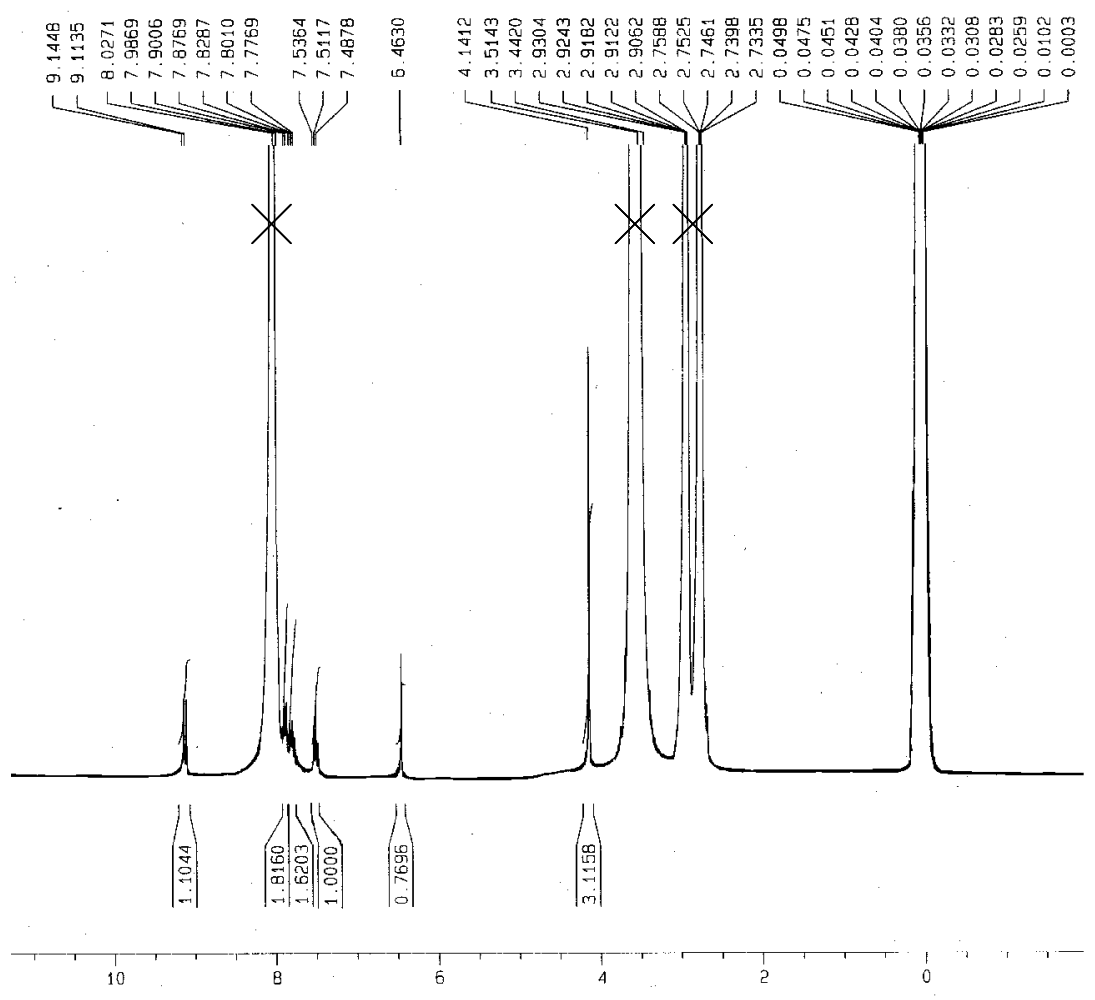

Figure S5. ${ }^{1} \mathrm{H}$ NMR spectrum of the croconaine dye $\mathbf{3 a}$ in DMF- $\mathrm{d}_{7}$.

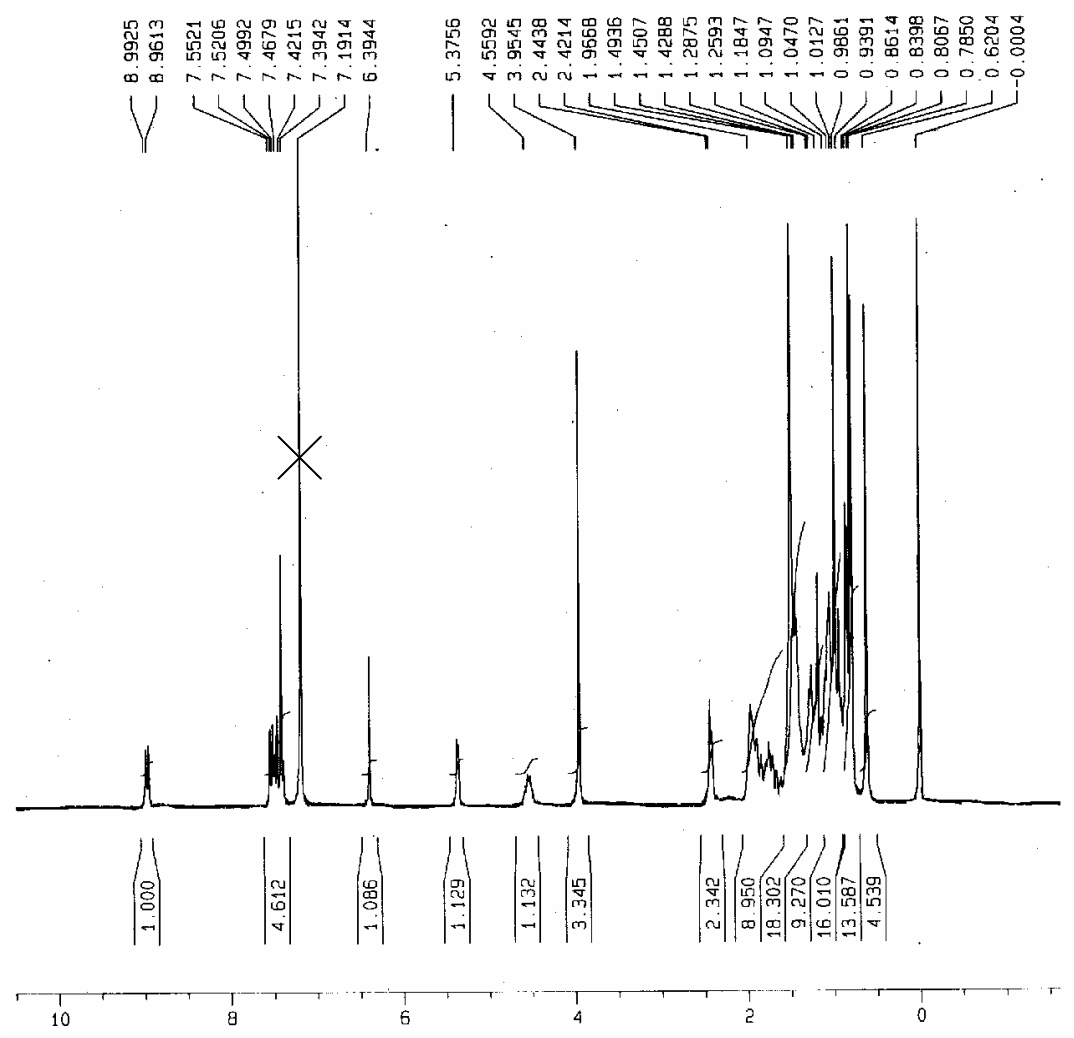

Figure S6. ${ }^{1} \mathrm{H}$ NMR spectrum of the croconaine dye $\mathbf{3 d}$ in $\mathrm{CDCl}_{3}$. 


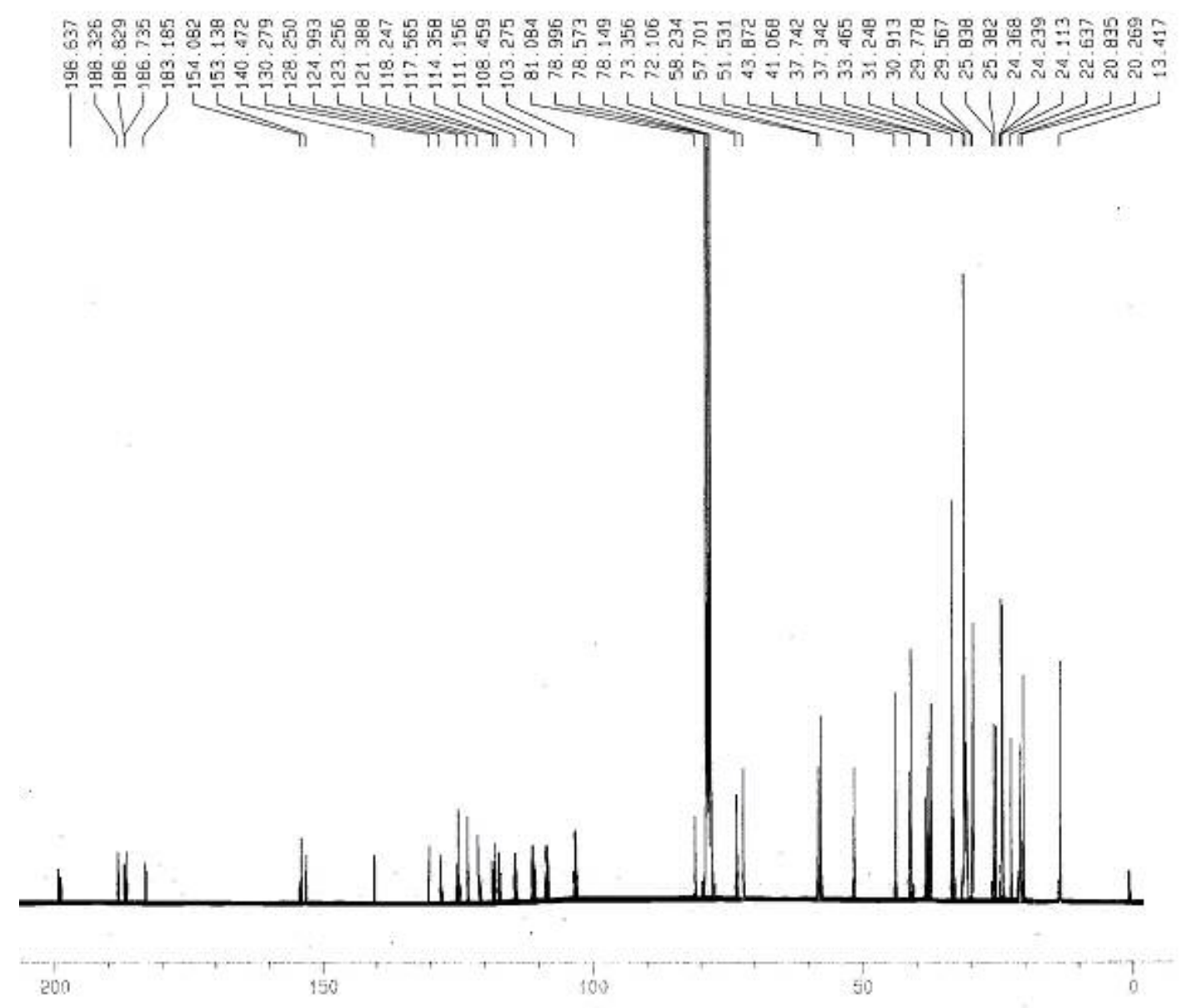

Figure S7. ${ }^{13} \mathrm{C}$ NMR spectra of croconaine dye $\mathbf{3 d}$ in $\mathrm{CDCl}_{3}$.

Table S1. Absorption properties of the croconaine dyes 3a-d. ${ }^{a}$

\begin{tabular}{ccc}
\hline Compound & Absorption, nm & $\varepsilon, \mathrm{M}^{-1} \mathrm{~cm}^{-1}$ \\
\hline $\mathbf{3 a}^{b}$ & 842 & $1.3 \times 10^{5}$ \\
$\mathbf{3 b}^{b}$ & 861 & $1.9 \times 10^{5}$ \\
$\mathbf{3 c}^{b}$ & 865 & $1.4 \times 10^{5}$ \\
$\mathbf{3 d}^{c}$ & 865 & $4.2 \times 10^{5}$ \\
${ }^{a}$ Average of more than 2 experiments. ${ }^{b}$ In DMF. ${ }^{c}$ In $\mathrm{CHCl}_{3 .}$. \\
\hline
\end{tabular}


A

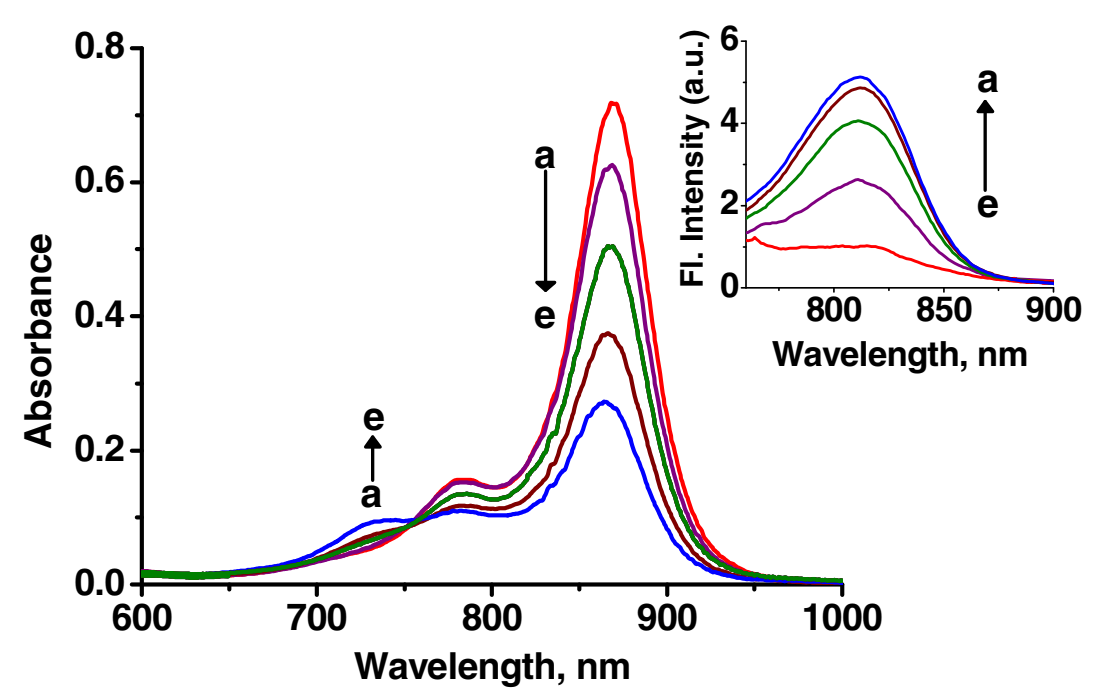

B

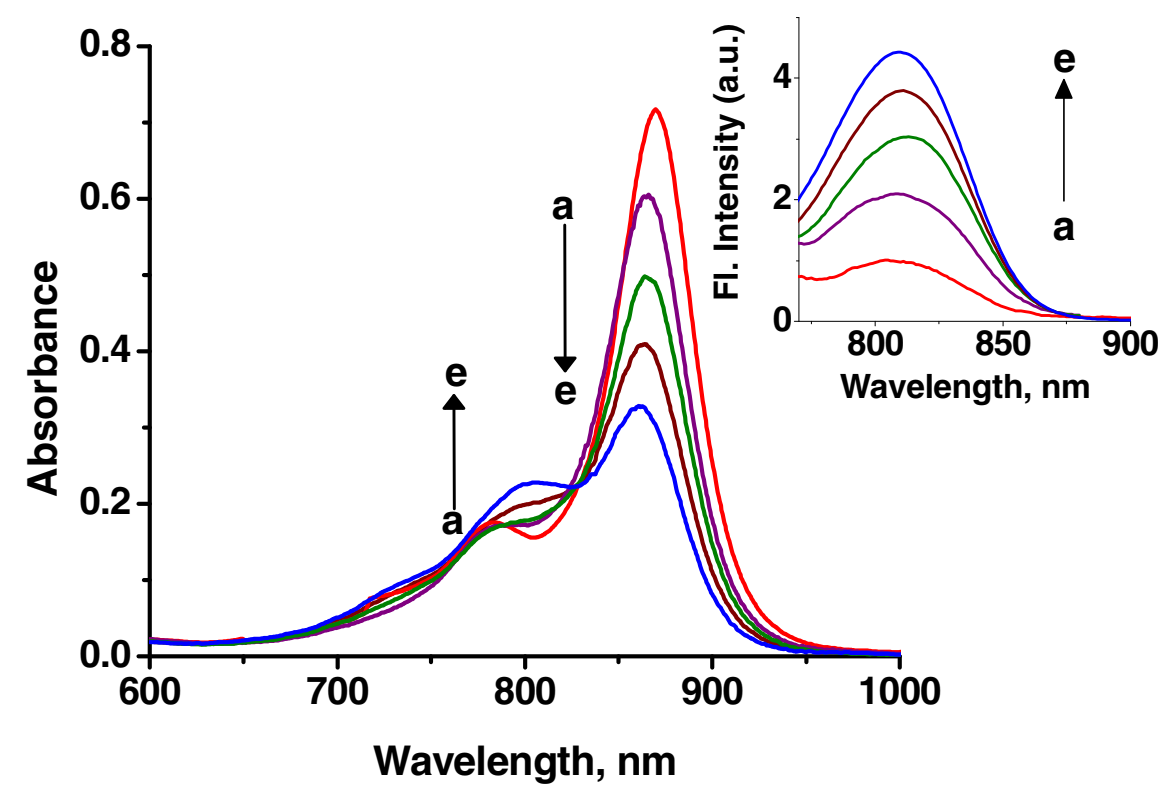

Figure S8. Changes in the absorption spectra of the dye 3d $(7.75 \mu \mathrm{M})$ in THF with the addition of metal ions. $\mathrm{M}^{\mathrm{n}+}:$ (A) $\mathrm{Hg}^{2+}$ and (B) $\mathrm{Mg}^{2+}$. $\left[\mathrm{M}^{\mathrm{n}+}\right]$ a) 0 , b) 0.94 , c) 1.88 , d) 2.82 and e) $3.87 \mu \mathrm{M}$. Inset shows the corresponding changes in fluorescence intensity. Excitation wavelength, $700 \mathrm{~nm}$. 


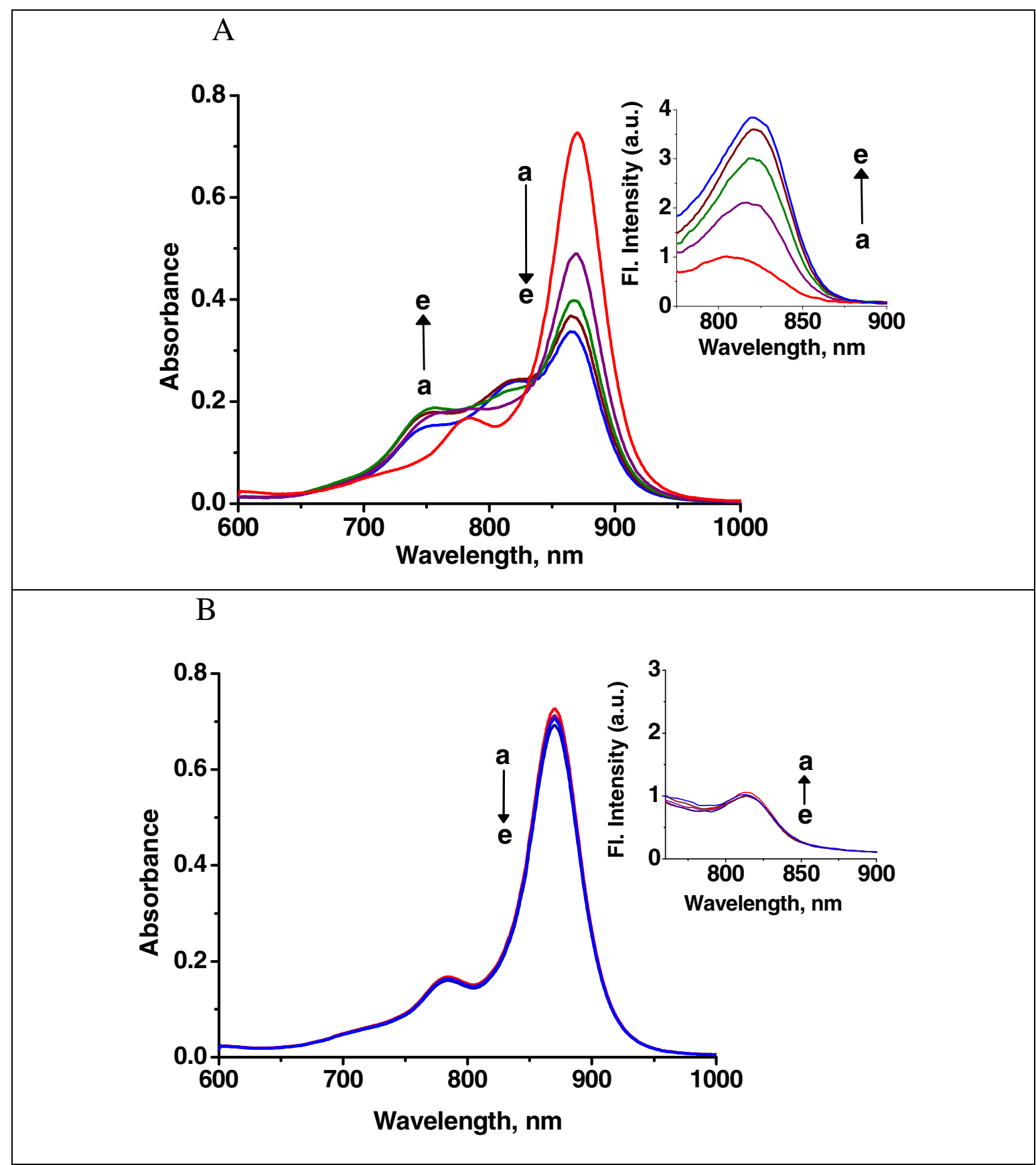

Figure S9. Changes in the absorption spectra of the dye 3d $(7.75 \mu \mathrm{M})$ in THF with the addition of metal ions. $\mathrm{M}^{\mathrm{n}+}$ : (A) $\mathrm{Ca}^{2+}$ and (B) $\mathrm{Na}^{+}$. $\left[\mathrm{M}^{\mathrm{n}+}\right]$ a) 0 , b) 0.94 , c) 1.88 , d) 2.82 and e) $3.87 \mu \mathrm{M}$. Inset shows the corresponding changes in fluorescence intensity. Excitation wavelength, $700 \mathrm{~nm}$. 